\title{
All-cause mortality and serum insulin-like growth factor I in primary care patients
}

\author{
N. Friedrich a,*, H. Schneider ${ }^{\text {b }}$, M. Dörr ${ }^{\text {c }}$, M. Nauck ${ }^{\text {a }}$, H. Völzke ${ }^{\text {d }}$, J. Klotsche ${ }^{\text {e }}$, C. Sievers ${ }^{\text {f }}$, \\ D. Pittrow ${ }^{\mathrm{g}}$, S. Böhler ${ }^{\mathrm{g}}$, H. Lehnert ${ }^{\text {h }}$, L. Pieper ${ }^{\mathrm{e}}$, H.-U. Wittchen ${ }^{\mathrm{e}}$, H. Wallaschofski ${ }^{\mathrm{a}, 1}$, \\ G.K. Stalla ${ }^{g, 1}$ \\ a Institute for Clinical Chemistry and Laboratory Medicine, Ernst Moritz Arndt University of \\ Greifswald, Germany \\ b Department of Internal Medicine, Department of Endocrinology, Ludwig-Maximilians-University of \\ Munich, Germany \\ c Department of Cardiology, Ernst Moritz Arndt University of Greifswald, Germany \\ d Institute for Community Medicine, Ernst Moritz Arndt University of Greifswald, Germany \\ e Institute of Clinical Psychology, Center of Clinical Epidemiology and Longitudinal Studies \\ (CELOS), Technical University Dresden, Germany \\ f Institute of Clinical Pharmacology, Technical University Dresden, Germany \\ g Max Planck Institute of Psychiatry, Munich, Germany \\ h 1st Dept. of Medicine, University of Luebeck, Germany
}

\begin{abstract}
Objective: Previous population-based studies provided conflicting results regarding the association of total serum insulin-like growth factor I (IGF-I) and mortality. The aim of the present study was to assess the relation of IGF-I levels with all-cause mortality in a prospective study.

Design: DETECT (Diabetes Cardiovascular Risk-Evaluation: Targets and Essential Data for Commitment of Treatment) is a large, multistage, and nationally representative study of primary care patients in Germany. The study population included 2463 men and 3603 women. Death rates were recorded by the respective primary care physician. Serum total IGF-I levels were determined by chemiluminescence immunoassays and categorized into three groups (low, moderate, and high) according to the sex- and age-specific 10th and 90th percentiles. Results: Adjusted analyses revealed that men with low [hazard ratio (HR) $1.70(95 \%$ confidence interval [CI] 1.05-2.73), $\mathrm{p}=0.03$ ] and high [HR 1.76 (95\% CI 1.09-2.85), $\mathrm{p}=0.02$ ] IGF-I levels had higher risk of all-cause mortality compared to men with moderate IGF-I levels. The specificity of low IGF-I and high IGF-I levels increased with lower and higher cut-offs, respectively. No such association became apparent in women.

Conclusions: The present study revealed a U-shaped relation between IGF-I and all-cause mortality in male primary care patients.
\end{abstract}

Keywords: IGF-I, Mortality, DETECT, Primary care

\section{Introduction}

Insulin-like growth factor I (IGF-I), which is mostly carried by IGF binding protein 3 (IGFBP-3), is generally accepted as a central mediator of endocrine and finally metabolic effects of the growth hormone $(\mathrm{GH})$. Less than $1 \%$ are available as free bioactive IGF-I and in epidemiological studies usually the total IGF-I levels are used. Both disorders of the GH/IGFI axis, GH deficiency (GHD) characterized by low IGF-I serum values as well as acromegaly 
accompanied by GH and finally IGF-I oversecretion, are related to increased morbidity [1] and mortality [2]. Several studies demonstrated that GHD is associated with altered lipid profile, insulin resistance, and glucose intolerance due to an increase of visceral fat mass [3]. Therefore GHD showed a strong correlation with the metabolic syndrome which might be a reason for the observed higher mortality in GHD patients [4,5]. Moreover, an increased mortality has been reported also in acromegaly mostly due to cardiovascular disease (CVD) and respiratory diseases [1]. Therefore a U-shaped association between all-cause mortality and GH/IGF-I might be assumed.

However, so far population-based studies investigating the relation of total IGF-I to mortality revealed conflicting results. The Rancho Bernardo Study [6] and the Framingham Heart Study [7] demonstrated inverse relations between IGF-I levels and ischemic heart disease mortality or all-cause mortality, respectively. A Danish study showed that high IGF-I levels were related to an increased all-cause mortality [8]. In contrast, two further population-based studies, the National Health and Nutrition Examination Survey (NHANES) [9] and the InChianti study [10] detected no association between IGF-I levels and mortality. The most likely reason for the conflicting results might be different sample sizes, definitions of outcome or confounders as well as different methods to measure IGF-I.

Unfortunately the above-mentioned studies did not consider the possible U-shape of the association between IGF-I and mortality. In a recent analyses of the Study of Health in Pomerania (SHIP) [11], we investigated low as well as high IGF-I levels in relation to allcause mortality and demonstrated that low IGF-I levels were related to an almost two-fold higher mortality risk in men but not women. No associations were detected for high IGF-I levels. Possible reasons for the lack of an association between high IGF-I values and all-cause mortality might be the relative low number of events and the fact that a relatively healthy setting were reflected in such a population-based study design.

In contrast to population-based studies, where much research on the association of IGF-I to mortality has been done, little is known about the potential effects of GH levels on mortality in primary care patients, which might be suffered more often from diseases. Therefore, the aim of the present study was to reinvestigate the association between low and high IGF-I levels and all-cause mortality in German primary care patients and comparing the obtained results with findings in the general German population [11]. We hypothesize that even though this setting comprise more diseased subjects than the general population, low IGF-I and possible high IGF-I levels are associated with an increased all-cause mortality.

\section{Materials and methods}

\subsection{Study design}

DETECT (Diabetes Cardiovascular Risk-Evaluation: Targets and Essential Data for Commitment of Treatment) is a large, multistage, and nationally representative study in Germany [12].On 16th and 18th September 2003 half a day, 3188 GPs completed a standardized assessment of the diagnostic and therapeutic profile of 55,518 unselected consecutive patients (59\% women and $41 \%$ men; over 17 years). All patients completed a questionnaire on demographic data, complaints, illness history, knowledge about selected diseases and attitude towards those. The initial physicians' response rate was $60.2 \%$, and further adjustments for nonresponse, regional distribution, and attrition were performed. In addition, a random subsample of 7519 patients participated in a more intensive standardized laboratory assessment. Of these patients, 6826 participated in a 1-year or 4-year follow-up, 
which was conducted in 2004 or 2007/2008, respectively. Another 279 subjects died during the follow-up period, and 693 subjects were lost to follow-up (Fig. 1). The last contact (last follow-up or day of death) was considered the end of that patient's follow-up. The comparison between subjects $(n=693)$ who were lost to follow-up and the remaining patients $(n=6826)$ revealed that the latter were, on average, 6 years older. However, no significant differences regarding the age- and sex-adjusted proportion of diabetes mellitus, ischaemic heart disease (IHD), liver, or renal diseases were found.

Of the 6826 subjects, eleven subjects were excluded due to missing information on time of death. Furthermore, 663 patients with missing data for IGF-I and 86 patients with missing data for used continuous confounders were also excluded. Valid data were available in 2463 men and 3603 women aged 18 to 95 years.

\subsection{Measurements}

For all patients, a comprehensive standardized clinical evaluation (patients' self-report and physicians' assessments) was performed. Information on diabetes mellitus, thyroid dysfunction, renal, liver, and ischemic heart disease (IHD) as well as height and weight were collected by physician-completed questionnaires. Body mass index [BMI=body weight (kg)/height [2] (m2)] was calculated. Smoking status and physical training were assessed by patients self-reports. Smoking was categorized into smokers and non-smokers. Patients who performed physical training for at least $2 \mathrm{~h}$ a week were classified as being physically active. Missing values in smoking status and physical activity were treated as separate group. To define acromegaly Schneider et al. [13] contacted 125 patients with IGF-I levels above the age- and sex-specific reference range. Of these 125 subjects, 7 patients had non-suppressed $\mathrm{GH}$ levels in an oral glucose tolerance test and were considered biochemically acromegalic.

Blood samples were collected and shipped by courier at room temperature within $24 \mathrm{~h}$ to the central laboratory. Upon arrival in the central laboratory, the samples were centrifuged immediately, and the serum was stored at $-20{ }^{\circ} \mathrm{C}$ until further processing. IGF-I was determined with an automated chemiluminescence system (Nichols Institute Diagnostics San Clemente, CA). The maximal intra- and interassay coefficients of variation were $5 \%$ and $7 \%$, respectively. Reagents and secondary standards were used as recommended by the manufacturer. In every 5-years age-group, IGF-I levels were categorized into three groups (low, moderate, and high) according to the sex-specific 10th and 90th percentiles of the study population [11]. In the DETECT study, mortality and incident events were recorded by the respective primary care physician.

\subsection{Statistical analysis}

Categorical data were expressed as percentages; continuous data were expressed as mean (standard deviation). Univariate analysis was performed with $\chi 2$ test for categorical variables and Mann-Whitney- U-test for continuous distributions. In a first step, age-adjusted restricted cubic splines with 3 knots were used to detect a possible non-linear dependency of the log hazard function on serum IGF-I levels. Based on these results in a second step, multivariable Cox proportional hazard regression models with age as timescale were run to assess the associations between low as well as high IGF-I levels and all-cause mortality separately for men and women. The models were adjusted for BMI, smoking and physical activity. Further comorbidities including diabetes, thyroid, renal, liver diseases, and IHD were tested as potential confounders. Comorbidities which led to a N5\% change in the estimate of interest remained in the models. Following this, in men the model was further adjusted for IHD and 
liver disease, whereas in women only liver disease was additionally included. The model assumption for the Cox proportional hazards regression model was checked with Schoenfeld residuals and $\log$ of the negative log of survival plots. Hazard ratios (HR) with $95 \%$ confidence intervals (CI) were calculated. A value of pb0.05 was considered statistically significant. Statistical analyses were performed with SAS 9.1 (SAS Institute Inc., Cary, NC, USA).

\section{Results}

\subsection{General characteristics}

In the study population, 241 men and 353 women were classified as subjects with low IGF-I or high IGF-I levels, respectively. Comparisons regarding general characteristics are presented in Table 1. In both sexes, subjects with low IGF-I levels had higher BMI and were more often affected by diabetes mellitus or liver diseases. Additionally, men with low IGF-I levels were more often smokers and women with low IGF-I levels were less often physically active and less often affected by thyroid disease than subjects with moderate hormone levels. No differences were found between subjects with high and moderate IGF-I levels in both men and women.

\subsection{Association of IGF-I levels with mortality}

During 19,129 person-years of follow-up, 5.6\% $(n=137)$ of men and $2.9 \%(n=106)$ of women died. In a first step, we performed Cox regression with restricted cubic splines to assess the functional form of the association between IGF-I and mortality. The results showed a Ushaped significant association in men but not women (Fig. 2). Results of univariate KaplanMeier survival analysis and multivariate Cox proportional hazard analyses using IGF-I categorizations are presented in Fig. 3 and Table 2, respectively. In univariate Kaplan- Meier survival analysis, low and high IGF-I levels were associated with a higher all-cause mortality compared to moderate IGF-I levels in men (log-rank test: pb0.01). Analysis of IGF-I quartiles also revealed this U-shaped association (data not shown). Multivariable Cox proportional hazard analyses (Table 2) confirmed these results and showed that low and high IGF-I levels were independent predictors of all-cause mortality in men. In women, no relation between IGF-I levels and all-cause mortality were revealed. The exclusion of 11 patients with acromegaly, anterior pituitary insufficiency, or a pituitary adenoma did not affect these results [men: low IGF-I: hazard ratio (HR) 2.15 (95\% confidence-interval (CI) 1.36-3.41), pb0.01; high IGFI: HR 2.24 (95\%-CI 1.40-3.57), pb0.01; women: data not shown]. In further analysis we tested different definitions of low and high IGF-I levels by varying cut-offs (age specific 15th-85th, 20th-80th, and 25th-75th percentiles). This analysis revealed increasing risks of all-cause mortality when lower IGF-I cut-offs were chosen to define low IGF-I and when higher IGF-I cut-offs to define high IGF-I in men (Fig. 4) but not women (data not shown). This finding confirmed the U-shaped association between IGF-I and mortality in men.

\section{Discussion}

The present study demonstrated a U-shaped association between IGF-I values and all-cause mortality considering data from a cohort of primary care patients in men but not women. Low and high IGF-I levels were related to a higher all-cause mortality compared to moderate IGF-I levels in men. The specificity of low IGF-I and high IGF-I levels increased with lower and higher cut-offs, respectively. These associations were not affected by acromegaly. For the first time, low as well as high IGF-I was identified as an independent risk factor for mortality in a 
single study population. In women, the number of cases and thus the power might be too low to detect an association between IGF-I and mortality. Further studies with higher number of cases in women are needed. Moreover, the follow-up time was between one and four years and the study might be a premature analysis for definitive determinants of mortality. Therefore, reverse causation (i.e. that low and high IGF-I levels are a consequence rather than a cause of factors contributing to increased mortality) cannot be ruled out with certainty. However we adjusted for or tested the effect of several diseases possibly associated with IGFI changes.

Former studies showed that either low or high IGF-I levels were related to mortality. The findings regarding low IGF-I levels and all-cause mortality are in good agreement with a recent analysis from SHIP demonstrating an almost two-fold higher risk of all-cause mortality in men with low IGF-I levels [11]. In both studies SHIP and DETECT, the association between IGF-I and all-cause mortality was only detectable in men but not in women. While these findings are in good agreement with a previous analysis of DETECT showing already sex-specific differences regarding the association of IGF-I with dyslipidemia and IHD, the specific mechanisms underlying this sex-specifity remain to be investigated [14]. Beside SHIP, also the Framingham Heart Study confirmed our present results and found that with increasing levels of IGF-I all-cause mortality was reduced [7], unfortunately no sex-specific analyses were performed. On the other hand, the Rancho Bernardo Study [6] and the NHANES [9] found no association between IGF-I levels and all-cause mortality. However regarding cardiovascular mortality, the Rancho Bernardo Study detected that a $40 \mathrm{ng} / \mathrm{ml}$ decrease in IGF-I level was associated with a significant increase of $38 \%$ in risk of IHD mortality [6]. Unfortunately, the Rancho Bernardo Study also did not perform sex-specific analyses to clarify if sex differences were present. A recent Danish study, however, found the opposite of a positive association between high IGF-I levels and mortality [8].

The observed association between low or high IGF-I serum concentration and mortality was not specific to a single aetiology. It is well established that acromegaly, a state accompanied by GH excess resulting in high IGF-I levels, and GHD characterized by low IGF-I serum values are associated with increased morbidity and mortality [2]. Therefore, the reported Ushaped association between IGF-I and mortality is not unexpected in our study population. Outside the both extreme states of IGF-I regulation, acromegaly or GHD, the IGF-I axis is associated to many subclinical risk factors or phenotypes with increased mortality. Low IGF-I levels were detected in adults with anorexia, chronic obstructive pulmonary disease, liver cirrhosis, hypothyroidism, type 1 diabetes, and osteoporosis [15]. Former studies showed that critical illness is also associated with low IGF-I levels [15] suggesting that low IGF-I serum concentrations are rather a marker of pre-existing disease, than an independent risk factor of mortality. Therefore we carefully adjusted for medical morbidity and other clinical parameters, and continued to find an association between low as well as high IGF-I serum concentration and mortality in men. On the other side high IGF-I levels were related to cancer, renal disease, and hyperthyroidism [15]. Due to the broad range of function, IGF-I has nearly influence on all tissues of the human body. It is involved in cell proliferation, differentiation, as well as function. Regarding cardiovascular disease and cancer as main causes of mortality varies biological actions of IGF-I were found. For example experimental investigations demonstrated that IGF-I induces hypertrophy of cardiomyocytes and thus participates in the development of left ventricular hypertrophy (LVH) [16,17]. It has been demonstrated that cardiac IGF-I mRNA increased in parallel with hypertension and the onset of LVH [16]. Furthermore, IGF-I stimulates endothelial cells to release NO and therefore may represent a regulator of the vascular tone, which in turn interacts with $\mathrm{LVH}[18,19]$. In terms of cancer, numerous patient studies revealed that high IGF-I levels were related to an 
increased risk of different cancer types. In cancer patients a higher expression of IGF-I and IGF-2 was found, which indicated that in addition to the mitogenic effect of IGFs on cancer cells, a possible role might exist for IGFs in tumor growth and progression [20]. However, population-based studies did not confirm the positive association between IGF-I and cancer. One possible molecular link between low IGF-I and mortality might be the interaction of the latter one and Forkhead box O (FOXO) transcription factors. These FOXO transcription factors induce cell growth arrest and apoptosis, which can be prevented by FOXO phosphorylation by the survival kinase AKT in response to growth factors like IGF-I [21]. In the absence of growth factors, FOXOs reside in the nucleus and up-regulate genes that inhibit the cell cycle, promote apoptosis and decrease oxidative stress [22]. Together, the IGF-I axis is complex and account for functional diversity and the exact mechanism by which IGF-I may cause an increased risk of death is currently not known, we propose IGF-I being a risk marker, rather than risk factor. A risk marker is not assumed to play an etiologic or direct causal role, but is mainly useful to improve our ability to predict risk. Therefore, further answers about causality and pathogenesis could be inferred from long-term observations which should be replicated in multiple cohorts.

The aim of our study was to reanalyze the association between IGF-I and all-cause mortality in a collective of primary care patients and to compare results with findings of a populationbased study (SHIP). Both studies were conducted in the same country with the same health care system, and a broad range of confounding factors was considered. One point of strength of the present study was the measurement of IGF-I levels by same assay system. Limitations might have arisen from the single point determination of IGF-I which was measured only at baseline, assuming constant hormone levels in the study population. However, none of the above-mentioned population-based studies performed multiple measurements of IGF-I $[6,7,9,10]$. Furthermore, we did not measure IGF binding protein 3, the main binding protein, which might have an influence on the association between IGF-I and mortality. A further limitation was the high numbers of subjects with missing data for IGF-I levels in DETECT. However, comparison between patient with and without IGF-I measurements revealed no significant differences regarding sex, age, weight, height, proportion of diabetes mellitus, IHD thyroid, liver, and renal diseases.

In conclusion, our analyses showed that men with low as well as high IGF-I levels have higher all-cause mortality in a cohort of primary care patients. These results suggest a Ushaped association between the GH/IGF-I system and mortality. 


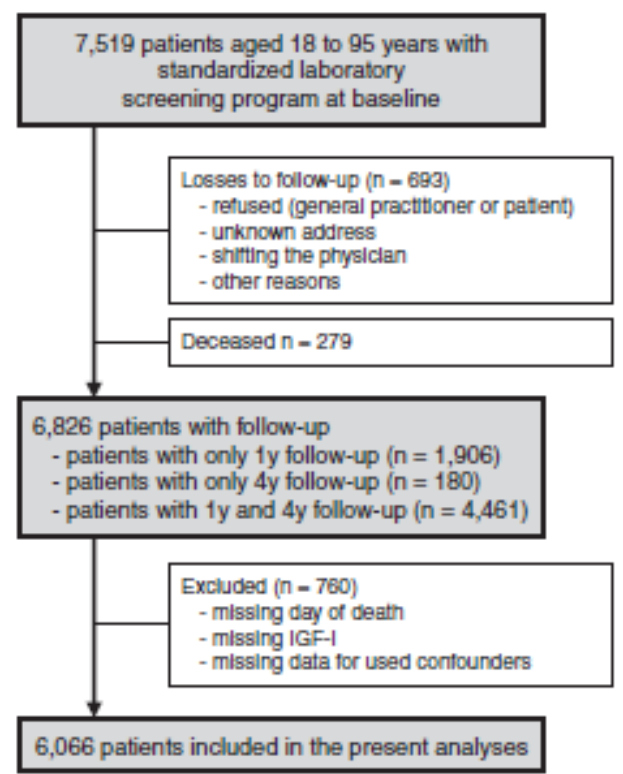

Fiz. 1. How diagram of study design of the prospective component of DETECr.

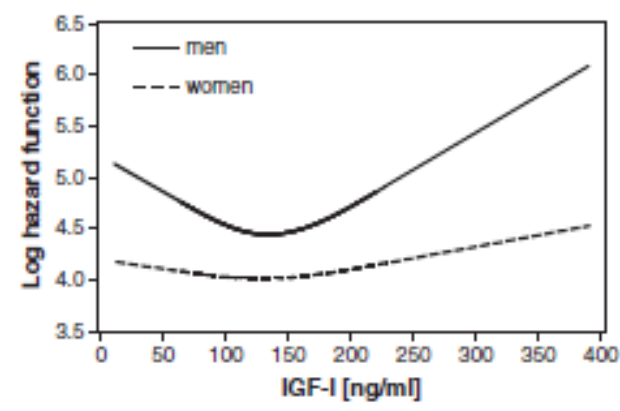

Fig. 2 Predicxed $\log$ hazard function for mortality as a function of 1 CF 1 in men $(-)$ and women (- - ). Results of age-adjusted Cox proportional hazard regression models with restricted cuhic splines.
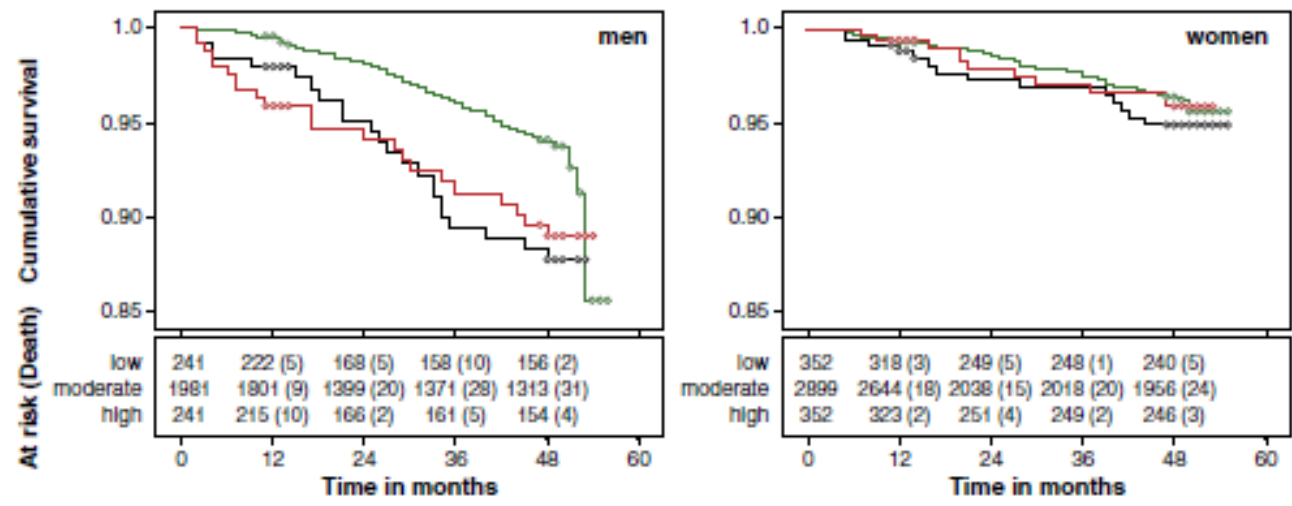

Fig 3. Survival aurves for all-cause morthlity by levels of insulin-like gowth factar 1 (1CF-l; — low, — moderat, and — high) in men and women. 


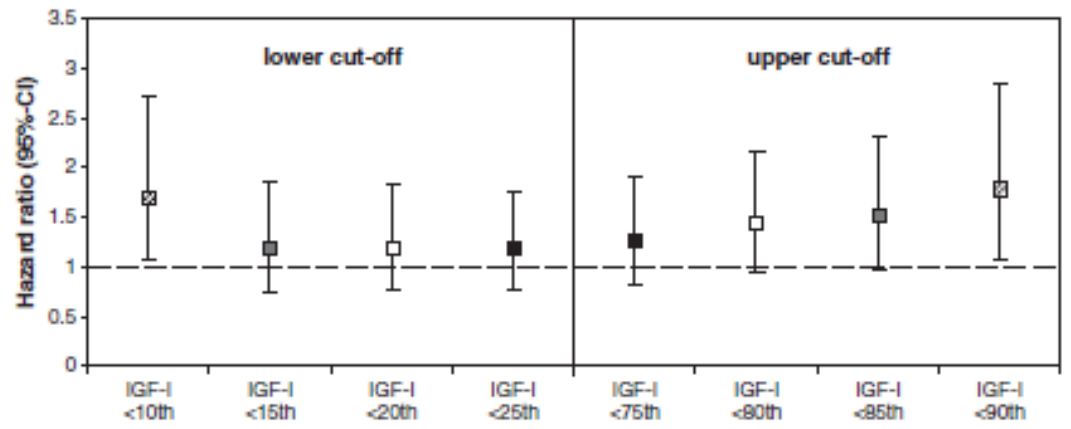

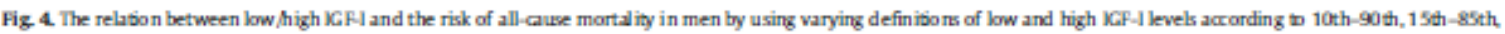

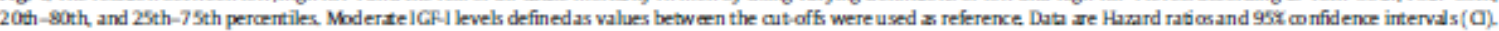

Table 1

Badine charateris bics stratified by sex and MGF-l levels

\begin{tabular}{|c|c|c|c|c|c|c|}
\hline \multirow[t]{2}{*}{ Charxteris bics } & \multicolumn{3}{|l|}{ Men with } & \multicolumn{3}{|l|}{ Women with } \\
\hline & $\begin{array}{l}\text { low ICFA- } \\
(\mathrm{N}=241)\end{array}$ & $\begin{array}{l}\text { Moderats XCFA } \\
(\mathrm{N}=1981)\end{array}$ & $\begin{array}{l}\text { High XCF-A } \\
(\mathrm{N}=241)\end{array}$ & $\begin{array}{l}\text { Low ICF-A } \\
(\mathrm{N}=352)\end{array}$ & $\begin{array}{l}\text { Moderats KCFH } \\
(\mathrm{N}=2 \mathrm{2099})\end{array}$ & $\begin{array}{l}\text { High XCF-A } \\
(\mathrm{N}=352)\end{array}$ \\
\hline Person-years & 745 & 6253 & 739 & 1117 & 9149 & 1126 \\
\hline Age (years) & $\operatorname{ses}(127)$ & $59.3(13.1)$ & $593(130)$ & $57.6(146)$ & $57.4(148)$ & 57.5 (146) \\
\hline \multicolumn{7}{|l|}{ Smoking $(x)$} \\
\hline Non-smokers & 65.6 & 76.1 & 76.4 & 73.3 & 740 & 744 \\
\hline Smokers & 28.6 & 170 & 145 & 17.6 & 17.1 & 168 \\
\hline Messing values & 5.8 & 69 & 9.1 & 91 & 90 & 88 \\
\hline \multicolumn{7}{|l|}{ Physially xtive (x) } \\
\hline$<2$ h/wedk & 307 & 24.4 & 27.8 & 37.8 & 30.4 & 31.0 \\
\hline$\geq 2$ h/week & 622 & 0.1 & 668 & 51.7 & 610 & 628 \\
\hline Messing values & 7.1 & 6.5 & 5.4 & 105 & 86 & 6.3 \\
\hline BMe $\left(\mathrm{kg} / \mathrm{m}^{2}\right)$ & $285(48)$ & $273(4.2)$ & $27.5(41)$ & $28.1(6.1)$ & 266 (5.1) & 26.1 (42) \\
\hline Dishetes mellitus $(\mathscr{x})$ & 299 & 22.1 & 224 & 221 & 139 & 16.5 \\
\hline Renal disease $(x)$ & 87 & 6.3 & 87 & 6.5 & 5.5 & $5 . A$ \\
\hline Liver disease ( $(x)$ & 16.2 & 49 & 33 & 57 & 2.7 & 28 \\
\hline $\operatorname{IHD}(x)$ & 199 & 20.3 & 245 & 11.9 & 93 & 125 \\
\hline Thyroid disease $(x)$ & 50 & 6.1 & 6.2 & 11.1 & 178 & 179 \\
\hline $\mathrm{KGH}(\mathrm{ng} / \mathrm{ml})$ & $62(17)$ & $130(34)$ & $227(56)$ & $62(17)$ & $126(37)$ & $230(\Theta 9)$ \\
\hline
\end{tabular}

IGF $=$ insulin-like growth factor 1 ; $\mathrm{MM}=$ body mass index; $1 \mathrm{HD}=$ ischenic heart disease. Continuous data are exp ressed a mean (standard deviation); nominal data are given as

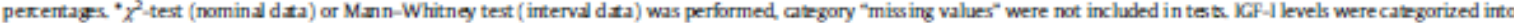
three levels according to the age- and sex-specific 10th and 90th percentiles

Table 2

Assodiation between MCF-1 levels and all-cause mortality.

\begin{tabular}{|c|c|c|c|c|}
\hline & \multicolumn{4}{|c|}{ ICFH levek (ref: moderate) } \\
\hline & \multicolumn{2}{|l|}{ Low } & \multicolumn{2}{|l|}{ High } \\
\hline & HR (95x-a) & $\mathrm{p}$ & HR (95E CI) & $\mathrm{p}$ \\
\hline \multicolumn{5}{|l|}{$\operatorname{Men}(N=2463)$} \\
\hline Unadjusted & $2.15(1.36 ; 3.41)$ & $<001$ & $216(1.35 ; 344)$ & $<001$ \\
\hline Fully adjusted ${ }^{\dagger}$ & $1.70(1.05 ; 273)$ & $a a_{3}$ & $1.76(109 ; 285)$ & 002 \\
\hline \multicolumn{5}{|c|}{ Wamer $(N=3603)$} \\
\hline Unadjusted & $1.47(0.83 ; 260)$ & a.19 & 1.11 (0.59; 209) & 074 \\
\hline Fully djusted ${ }^{\dagger}$ & $135(\alpha, 76 ; 241)$ & a.31 & $1.21(0.64 ; 228)$ & 0.56 \\
\hline
\end{tabular}

ICFM = insulin-like grow th f $x$ tor l; $\mathrm{BM}$ = body mass index; CVD = cardiovascular disesse; $H R=$ haz:ard rabia; $\mathrm{Q}=$ confidence interval.

$\dagger$ The full model were aljusted for BML smoking, physial activity, live diseze and ischenic heart disese (only in men). Age was used as timescale. MCFA levds were cazgorized int three groups according to the age- and sex-specific 10th and 90th perentiles

\section{Acknowledgment}

Diabetes cardiovascular risk-evaluation: targets and essential data for commitment of treatment (DETECT) is a cross-sectional and prospective-longitudinal, nationwide clinical epidemiological study. Principal investigator: Prof. Dr. H.-U. Wittchen; Staff members: Dipl.Psych. L. Pieper, Dipl.-Math. J. Klotsche, Dipl.-Psych. T. Eichler, Dr. H. Glaesmer, E. Katze, Dipl.-Psych. A. Bayer, Dipl.-Psych. A. Neumann. Steering Committee: Prof. Dr. H. Lehnert 
(Magdeburg, Coventry), Prof. Dr. G.K. Stalla (Munich), Prof. Dr. M.A. Zeiher (Frankfurt); Advisory Board: Prof. Dr. W. März (Graz/Heidelberg), Prof. Dr. S. Silber (Munich), Prof. Dr. Dr. U. Koch (Hamburg), PD Dr. D. Pittrow (Munich, Dresden), and Professor Dr. M.

Wehling (Mannheim).

This work is part of the research project Greifswald Approach to Individualized Medicine (GANI_MED). The GANI_MED consortium is funded by the Federal Ministry of Education and Research and the Ministry of Cultural Affairs of the Federal State of Mecklenburg West Pomerania (03IS2061A).

\section{References}

[1] A. Colao, D. Ferone, P. Marzullo, G. Lombardi, Systemic complications of acromegaly: epidemiology, pathogenesis, and management, Endocr. Rev. 25 (2004) 102-152.

[2] O.M. Dekkers, N.R. Biermasz, A.M. Pereira, J.A. Romijn, J.P. Vandenbroucke, Mortality in acromegaly: a metaanalysis, J. Clin. Endocrinol. Metab. 93 (2008) 61-67.

[3] N. Mathioudakis, R. Salvatori, Adult-onset growth hormone deficiency: causes, complications and treatment options, Curr. Opin. Endocrinol. Diabetes Obes. 15 (2008) 352-358.

[4] K. Stochholm, J. Christiansen, T. Laursen, C.H. Gravholt, Mortality and reduced growth hormone secretion, Horm. Res. 68 (Suppl 5) (2007) 173-176.

[5] K. Stochholm, C.H. Gravholt, T. Laursen, et al., Mortality and GH deficiency: a nationwide study, Eur. J. Endocrinol. 157 (2007) 9-18.

[6] G.A. Laughlin, E. Barrett-Connor, M.H. Criqui, D. Kritz-Silverstein, The prospective association of serum insulin-like growth factor I (IGF-I) and IGF-binding protein-1 levels with all cause and cardiovascular disease mortality in older adults: the Rancho Bernardo Study, J. Clin. Endocrinol. Metab. 89 (2004) 114-120.

[7] R. Roubenoff, H. Parise, H.A. Payette, et al., Cytokines, insulin-like growth factor 1, sarcopenia, and mortality in very old community-dwelling men and women: the Framingham Heart Study, Am. J. Med. 115 (2003) 429-435.

[8] M. Andreassen, I. Raymond, C. Kistorp, P. Hildebrandt, J. Faber, L.O. Kristensen, IGF1 as predictor of all cause mortality and cardiovascular disease in an elderly population, Eur. J. Endocrinol. 160 (2009) 25-31.

[9] S. Saydah, B. Graubard, R. Ballard-Barbash, D. Berrigan, Insulin-like growth factors and subsequent risk of mortality in the United States, Am. J. Epidemiol. 166 (2007) 518-526.

[10] M. Maggio, F. Lauretani, G.P. Ceda, et al., Relationship between low levels of anabolic hormones and 6-year mortality in older men: the aging in the Chianti Area (InCHIANTI) study, Arch. Intern.

Med. 167 (2007) 2249-2254.

[11] N. Friedrich, R. Haring, M. Nauck, et al., Mortality and serum insulin-like growth factor (IGF)-I and IGF binding protein 3 concentrations, J. Clin. Endocrinol. Metab. 94 (2009) 1732-1739.

[12] H.U. Wittchen, H. Glaesmer, W. Marz, et al., Cardiovascular risk factors in primary care: methods and baseline prevalence rates - the DETECT program, Curr. Med. Res. Opin. 21 (2005) 619-630.

[13] H.J. Schneider, C. Sievers, B. Saller, H.U. Wittchen, G.K. Stalla, High prevalence of biochemical acromegaly in primary care patients with elevated IGF-1 levels, Clin. Endocrinol. (Oxf.) 69 (2008) $432-435$.

[14] H.J. Schneider, J. Klotsche, B. Saller, et al., Associations of age-dependent IGF-I SDS with cardiovascular diseases and risk conditions: cross-sectional study in 6773 primary care patients, Eur. J. Endocrinol. 158 (2008) 153-161.

[15] A. Juul, Serum levels of insulin-like growth factor I and its binding proteins in health and disease, Growth Horm. IGF Res. 13 (2003) 113-170.

[16] T.J. Donohue, L.D. Dworkin, M.N. Lango, et al., Induction of myocardial insulin-like growth factor-I gene expression in left ventricular hypertrophy, Circulation 89 (1994) 799-809.

[17] J. Ren, L. Jefferson, J.R. Sowers, R.A. Brown, Influence of age on contractile response to insulinlike growth factor 1 in ventricular myocytes from spontaneously hypertensive rats, Hypertension 34

(1999) 1215-1222. 
[18] H. Tsukahara, D.V. Gordienko, B. Tonshoff, M.C. Gelato, M.S. Goligorsky, Direct demonstration of insulin-like growth factor-I-induced nitric oxide production by endothelial cells, Kidney Int. 45 (1994) 598-604.

[19] M.F. Walsh, M. Barazi, G. Pete, R. Muniyappa, J.C. Dunbar, J.R. Sowers, Insulin-like growth factor I diminishes in vivo and in vitro vascular contractility: role of vascular nitric oxide, Endocrinology 137 (1996) 1798-1803.

[20] A.A. Samani, S. Yakar, D. LeRoith, P. Brodt, The role of the IGF system in cancer growth and metastasis: overview and recent insights, Endocr. Rev. 28 (2007) 20-47.

[21] H. Huang, D.J. Tindall, Dynamic FoxO transcription factors, J. Cell Sci. 120 (2007) 2479-2487. [22] J.H. Paik, R. Kollipara, G. Chu, et al., FoxOs are lineage-restricted redundant tumor suppressors and regulate endothelial cell homeostasis, Cell 128 (2007) 309-323. 